'Departamento de Salud Pública, Facultad de Medicina, Universidad Católica del Norte. Coquimbo, Chile. aBiólogo, MSc.

bInterno de Medicina, Facultad de Medicina, Universidad Católica del Norte. Coquimbo, Chile.

El manuscrito no tuvo reparos éticos por parte del Comité Ético Científico de la Facultad de Medicina, Universidad Católica del Norte.

Trabajo no recibió financiamiento. Los autores declaran no tener conflictos de interés.

Recibido el 11 de marzo de 2019 , aceptado el 2 de abril de 2020 .

Corespondencia a: Domingo Lancellotti Larrondo 1281. Coquimbo, Chile. dlancell@ucn.cl

\section{Evaluación de la metodología Team Based Learning en cursos de estadística para estudiantes de medicina}

\author{
DOMINGO LANCELLOTTI ${ }^{1, \mathrm{a}}$, ALEJANDRO ABARCA ${ }^{1, \mathrm{a}}$, \\ JAVIERA JORQUERA ${ }^{\mathrm{b}}$, CAMILA LOBOS ${ }^{\mathrm{b}}$, \\ DIEGO AGUILERA ${ }^{\mathrm{b}}$, NELLY SÁNCHEZ
}

\section{Evaluation of team-based learning methodology for teaching statistics to medical students}

\begin{abstract}
Background: Team-Based Learning methodology (TBL) is used in medicine teaching in different clinical and basic fields, but seldom in statistics courses. Aim: To compare the performance of students taught using TBL with those receiving the traditional lecture modality. Materials and Methods: The grades and percentage of correct answers obtained in exams written during the statistics course of students that were taught without TBL (years 2009-2011) or using this methodology (years 2013-2016) were compared. Z Test for one mean (Milestone 1) and Student's t test for two independent means assuming equal variances/ unequal variances (Milestone 2) were evaluated. The mean and minimum grades and the percentage of grades below the flunking threshold were also evaluated. Students' assessment of the methodology was also recorded. Results: The percentage of correct answers was $94.2 \%$ and $94 \%$ among students taught with and without TBL methodology, respectively (Milestone 1), achieving a similar performance independent of the teaching strategy. The global percentage of correct answers of Milestone 2 increased from $75.3 \%$ to $89.5 \%(P<0.001)$ among students using TBL. In $75.0 \%$ of questions, the percentage of correct answers increased with TBL. Likewise, there was an increase in mean grades increased from 5.4 to $6.2(P<0.05)$ and mean minimum grades from 2.8 to $3.7(P=0.05)$. The proportion of grades below the flunking threshold decreased from $9.9 \%$ to $4.4 \%(P=0.054)$. Almost all students evaluated TBL methodology positively. Conclusions: Students taught using TBL methodology performed better in the statistics course evaluation than students attending standard lectures in statistical analysis of greater complexity. TBL was well evaluated.
\end{abstract}

(Rev Med Chile 2020; 148: 488-495)

Key words: TBL; Biostatistical course; Medical Student.
41 estudio de la estadística es parte del currículum de pregrado de la carrera de medicina, consecuencia de la importancia que ha alcanzado la investigación científica en la formación del médico ${ }^{1-3}$ pero, principalmente, por tributar al objetivo de situar la práctica de la medicina sobre una sólida base científica ${ }^{4}$.
Teniendo como evidencia que el aprendizaje activo mejora el rendimiento del estudiante ${ }^{5}$, tácticas docentes basadas en el trabajo colaborativo han desplazado a la clase magistral ${ }^{6-8}$. Más aún, como las estrategias docentes centradas en el estudiante y llevadas a cabo con trabajo colaborativo se traducen en un aprendizaje más significativo para el 
estudiante, metodologías tales como el Team Based Learning (TBL) o Aprendizaje Basado en Equipos, han logrado gran popularidad ${ }^{9,10}$. El TBL permite trabajar con múltiples grupos pequeños (de cinco a ocho estudiantes por grupo) en un sólo salón de clases y ser dirigidos por un único instructor, lo que representa una ventaja frente a otras estrategias de aprendizaje activo al no incurrir en costos mayores que una clase magistral como, por ejemplo, sí lo hace el Aprendizaje Basado en Problemas (ABP) que demanda un instructor para cada grupo de estudiantes.

Básicamente, la metodología TBL consta de: (i) una lectura previa (estudio) que realiza el estudiante en horas no-presenciales, material que es preparado por el profesor; (ii) desarrollo de una prueba individual de entrada (de opción múltiple y selección única); (iii) desarrollo de la misma prueba en forma grupal, grupos que se conforman al azar en cada sesión; (iv) revisión de la prueba con derecho a apelación; $(v)$ desarrollo de una microclase que cierra el círculo enseñanza-aprendizaje; y ( $v i)$ una actividad de aplicación que comprende desarrollo de situaciones (ejercicios) ${ }^{11,12}$. En resumen, el TBL desafía al estudiante a ser más proactivo (la clase requiere una preparación previa), promueve el aprendizaje colaborativo (enseñanza entre pares) y mejora el pensamiento crítico (hay espacio para discutir y defender las soluciones).

Esta metodología fue desarrollada a fines de los 70's por Larry Michaelsen en un curso de Negocios en la Universidad de Oklahoma (EE. UU. $)^{13}$, pero ha sido ampliamente utilizada en la enseñanza de la medicina y en las ciencias de la salud tanto en la cátedra, taller, laboratorio y en la clínica ${ }^{9,10,14,15}$, agregándole innovaciones como el uso de escenas simuladas transmitidas en vivo y/o pregrabadas ${ }^{16}$, uso de pacientes reales en vez de escenarios escritos ${ }^{17}$, puesta en práctica en ambiente virtual ${ }^{18,19}$ o llevada a cabo como estrategia híbrida con $\mathrm{ABP}^{20,21}$. Si bien el trabajo colaborativo es utilizado ampliamente en la enseñanza de la estadística que en mayor o menor grado incluye elementos de la metodología TBL, son escasos los estudios que reportan el uso del TBL en cursos de estadística en las ciencias de la salud ${ }^{22-24}$.

Este estudio evalúa los resultados del uso de la metodología TBL en la enseñanza de la estadística en la carrera de medicina, comparando el desempeño de los estudiantes en cuatro años de docencia con metodología TBL versus el desempeño de estudiantes en años previos bajo modalidad de clase magistral.

\section{Metodología}

Los contenidos de estadísticas en la carrera de medicina de la Universidad Católica del Norte (UCN) son impartidos en las asignaturas Metodología de la Investigación I y II (MI-I y MI-II, respectivamente). Se implementaron 13 actividades de aprendizaje bajo modalidad TBL, seis en MI-I (segundo semestre) y siete en MI-II (tercer semestre), abarcando desde la estadística descriptiva hasta la estadística inferencial bivariada. Cada sesión de TBL reemplazó una clase magistral y, por tanto, mantuvo los objetivos de aprendizaje y el número total de clases. Se trabajó con grupos de 5 a 6 estudiantes. El material de lectura para cada sesión de TBL correspondió a una transcripción de las diapositivas de años anteriores, explicando paso a paso el desarrollo de cada análisis y prueba estadística y respectivos ejemplos. Luego de cuatro años de enseñanza bajo modalidad TBL, se estableció una serie de hitos a evaluar, eligiendo dos de ellos dada su relevancia en el abordaje de las pruebas estadísticas. El Hito 1 , prueba Z para una media (quinto TBL en MI-I), introduce al estudiante a la inferencia estadística, clase precedida por un TBL que aborda la distribución normal, tabla $\mathrm{Z}$ y correspondiente sesión de ejercicios. El Hito 2, prueba t de Student para dos medias independientes suponiendo varianzas iguales vs varianzas desiguales (primer TBL en MI-II), representa un salto de complejidad al incluir una tercera prueba estadística para establecer la homogeneidad de las varianzas -la prueba $\mathrm{F}$ - que supedita el uso de la prueba t correspondiente. El TBL es realizado en la segunda semana del semestre, precedido por una clase magistral donde se aborda la lectura de la tabla $t$, la prueba $t$ de Student para una media y prueba $t$ de Student de dos muestras no-independientes. Cada hito corresponde a un caso que es evaluado en la prueba de cátedra. Un caso va seguido de preguntas que, para los respectivos hitos, evalúa conceptos, planteamiento de hipótesis nula vs hipótesis alternativa, valor crítico, cálculo de la prueba estadística e interpretación, más grados de libertad para las pruebas $t, t^{\prime}$ y prueba F. Ejemplos de "Casos Tipo" se muestran en la Tabla 1. En 
estos, la respuesta de una pregunta no condiciona la respuesta de una siguiente, por tanto, la conclusión que se llega del análisis de una pregunta no está influida por la valoración realizada a otras preguntas.

El desempeño de los estudiantes bajo modalidad TBL se evaluó comparando el porcentaje de acierto (PA) logrado en preguntas de pruebas de cátedra sin TBL (años 2009-2011) vs el PA logrado en las respectivas preguntas de la correspondiente prueba de cátedra con TBL (años 2013-2016). La comparación fue pregunta por pregunta y total preguntas (global). También se comparó el promedio de notas, el promedio de nota mínima y el porcentaje de notas $<4,0$ de las respectivas pruebas de cátedra donde se evaluó cada hito sin versus con TBL. El análisis estadístico incluyó Tabla de Contingencia de Chi-cuadrado (para variables cualitativas) y prueba t de Student para dos muestras independientes previa verificación de las varianzas (para variables cuantitativas), todas a un $\alpha=0,05$. El tamaño de muestra para el Hito 1 (número de estudiantes que rindieron pruebas) fue de 152 vs 215 para sin TBL vs con TBL, y para el Hito 2 de 161(106) vs 226 respectivamente. El $n$ (106) del Hito 2 se debe a que se excluyó del análisis preguntas del año 2009 porque tenían una construcción distinta. Todas las preguntas fueron de opción múltiple y selección única.

Finalmente, se pesquisó la valoración que los estudiantes hacían de la metodología TBL, solicitándoles la opinión a los estudiantes de MI-I en dos promociones distintas. Se utilizó la encuesta que año a año se aplica al final del semestre en las distintas asignaturas de la Facultad de Medicina de la UCN, y es la retroalimentación que los docentes tienen de su desempeño. Esta encuesta es semi-estructurada y consta de 27 preguntas sobre metodología docente (7), pertinencia curricular (4), proceso de evaluación (6), aspectos administrativos (6) y vínculo estudiante-profesor (4), con un espacio al final donde los estudiantes pueden libremente hacer comentarios sobre la asignatura. A los estudiantes se les informa que la encuesta es anónima y voluntaria, como también los comentarios que en ella se pueden hacer, sin embargo, en ambas ocasiones se les solicitó expresamente que se refirieran a la metodología TBL dada la relevancia que tenía para la evaluación que el equipo docente realizaba a la metodología. Estos

\section{Tabla 1. "Casos Tipo" y respectivos saberes a evaluar en la prueba de cátedra}

\section{Caso Tipo para el Hito 1 (Prueba Z):}

A un grupo de pacientes hipertensos se les administra un medicamento por un período de tiempo y se espera que reduzca la presión arterial sistólica (PAS) a niveles normales (PAS por debajo de $140 \mathrm{~mm} \mathrm{Hg}$ ). Las respectivas Hipótesis Nula vs Hipótesis Alternativa (Ho) que el estudiante debe identificar y probar son $\mathrm{H}_{0} \geq 140$ vs $\mathrm{H}_{\mathrm{A}}<140$, siendo el valor crítico de la prueba (vc) a un $\alpha=0,05$ de $-1,64$. Luego, el estudiante enfrenta un set de datos, por ejemplo $n=10$, debiendo calcular la media de la muestra y obtener el $Z_{\text {calculado }}(\mathbf{C a l Z})$ considerando una $\mu=140$ y un valor de $\sigma$ que se le entrega. La conclusión que debe llegar el estudiante respecto a la $\mathrm{H}_{0}(\mathbf{C H o})$ es "se rechaza la $\mathrm{H}_{0}$ si el $Z_{\text {calculado }}$ es menor que $-1,64$ ", mientras que la interpretación de la prueba (IZ) es "si se rechaza $\mathrm{H}_{0}$ se concluye que el medicamento reduce significativamente la PAS en pacientes hipertensos, alcanzado niveles normales". Para no condicionar las respuestas de $\mathbf{C H o}$ e IZ, las respectivas preguntas consideran valores ficticios, lo que está debidamente informado.

\section{Caso Tipo para el Hito 2 (prueba de la t de Student):}

Se prueban 2 drogas $\left(D_{1}\right.$ y $\left.D_{2}\right)$ para el manejo de la PAS en pacientes hipertensos asignados al azar a cada tratamiento; se espera que las drogas difieran respecto a los valores de la PAS. Los conceptos a evaluar (Cp) corresponden a uso de prueba $Z$ vs prueba $t$, muestras independientes vs no-independientes y homocedasticidad. Las respectivas Ho son $H_{0}: D_{1}=D_{2}$ vs $H_{A}$ : $D_{1} \neq D_{2}$. Luego, se entregan dos sets de datos, por ejemplo, $n_{1}=7$ y $n_{2}=6$, para que el estudiante obtenga un $t_{\text {calculado }}$ para una prueba suponiendo varianzas iguales (Calt); como información adicional se entrega las desviaciones estándar $\left(S_{1}\right.$ y $\left.S_{2}\right)$. Se le pide al estudiante que obtenga los grados de libertad ( $\mathbf{g l}$ ) que para el ejemplo son $v=11$, y el respectivo valor crítico (vc) a un $\alpha=0,05$, que para un $v=11$ el estudiante debe elegir la alternativa $t_{0,05(2), 11}=-2,201$ y 2,201. La interpretación de la prueba (It) es "si se rechaza la $\mathrm{H}_{0}$ las drogas presentan diferencias significativas en el manejo de la PAS" y, refiriéndose a las medias, el estudiante debe indicar con qué droga se logró el menor valor promedio de la PAS. Luego, debe obtener el valor de la prueba $\mathrm{F}$ (CaIF) e interpretar la prueba (IF), por ejemplo, al enfrentarlos a un $\mathrm{F}_{\text {calculado }}>\mathrm{F}_{\text {crítico }}$ el estudiante debe responder que "las varianzas son significativamente distintas y lo que corresponde realizar es la prueba de la $t$ de Student suponiendo varianzas desiguales". Para no condicionar las respuestas de vc, It e IF, las respectivas preguntas consideran datos ficticios; aquí, los estudiantes también son informados debidamente de esos valores ficticios. 
comentarios son los que se incluyen en este estudio. El análisis se limitó en identificar, agrupar y cuantificar los comentarios sobre la metodología TBL. No se consideró la evaluación por pares al observar conductas subjetivas.

Los estudiantes cursaban el primer (MI-I) y segundo (MI-II) año de medicina, todos por primera vez. El 49,4\% fueron mujeres, sin diferencias significativas entre sin TBL vs con TBL $(p=0,446)$. El docente de la cátedra fue el mismo en ambos períodos.

\section{Resultados}

\section{Hito 1}

El PA del global alcanzó 94,2\% y 93,9\% en los períodos sin TBL vs con TBL, respectivamente $(p=0,833)$, sin diferencias significativas en ninguna de las preguntas $(p \geq 0,717)$. Los mayores PA fueron registrados en vc, con $97,4 \%$ y $96,3 \%$, y menores en IZ, con $89,5 \%$ y $91,2 \%$, respectivamente, diferencias que sólo fueron significativas en $\sin$ TBL $(\mathrm{p}=0,016)$ (Figura 1). Para las pruebas de cátedra sin y con TBL el promedio de notas fue 6,2 y $6,3(\mathrm{p}=0,383)$, el promedio de nota mínima de 3,8 y $3,5(\mathrm{p}=0,263)$ y porcentajes de notas $<4,0$ de $3,9 \%$ y $3,7 \%(p=0,875)$, respectivamente. Esta prueba también evaluó la prueba $Z$ para una y para dos proporciones.

\section{Hito 2}

Bajo modalidad TBL, el PA del global aumentó de $75,3 \%$ a $89,5 \%(\mathrm{p}<0,001)$, con aumentos

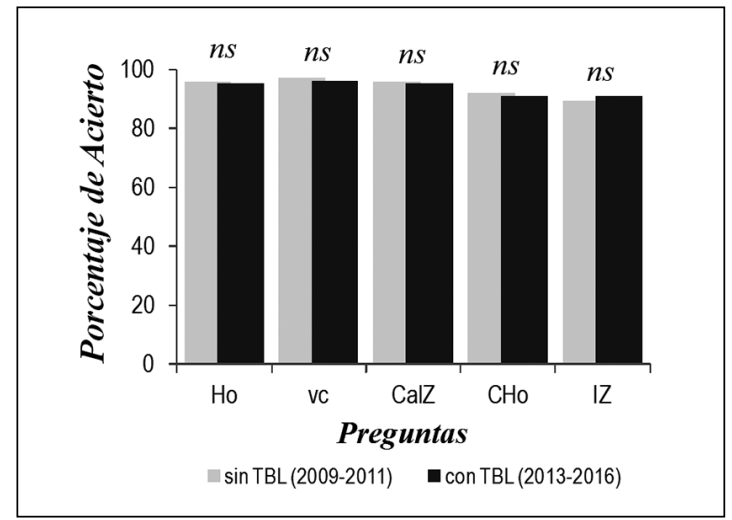

Figura 1. Porcentaje de acierto para las preguntas del Hito 1, períodos sin y con aprendizaje bajo modalidad TBL. Abreviaciones como en Tabla 1. (ns = no significativo). significativos en 6 de las 8 preguntas incluyendo It e IF (Figura 2). Los mayores aumentos se registraron en Ho y vc, con 29,8 y 22,0 puntos porcentuales, respectivamente, ambas preguntas con los menores PA en sin TBL. No se observaron diferencias significativas en gl y Calt, el primero mostrando uno de los mayores PA en ambos períodos y el segundo uno de los menores $\mathrm{PA}$ en con TBL $(79,6 \%)$ (Figura 2). De sin a con TBL, el promedio de notas aumentó significativamente de 5,4 a $6,2(p=0,005)$, aumentó significativamente el promedio de nota mínima de 2,8 a 3,7 $(\mathrm{p}=0,047)$, disminuyendo las notas $<4,0$ de $9,9 \%$ a $4,4 \%(\mathrm{p}=0,054)$, respectivamente. La prueba también evaluó la prueba $t$ de Student para una muestra y para dos muestras emparejadas.

\section{Valoración de la Metodología TBL}

De los 88 estudiantes que respondieron la encuesta, 61 hicieron comentarios a la metodología TBL, $59(96,7 \%)$ de ellos a favor (Tabla 2). De los estudiantes que mostraron su satisfacción, 70,5\% la definió como una estrategia facilitadora del aprendizaje. El 24,6\% valoró el estudio individual previo a la clase, mientras que $42,6 \%$ explicitó la ventaja de la retroalimentación grupal y/o por el profesor. Otros comentarios valoraron la posibilidad de trabajar con compañeros de curso que de otra manera nunca lo hubieran hecho, lo apropiado del material de lectura y el acceso a su nota durante la misma sesión. Hubo 2 comentarios en contra de la metodología que apuntaron a la necesidad de mejorar el material de lectura (Tabla 2).

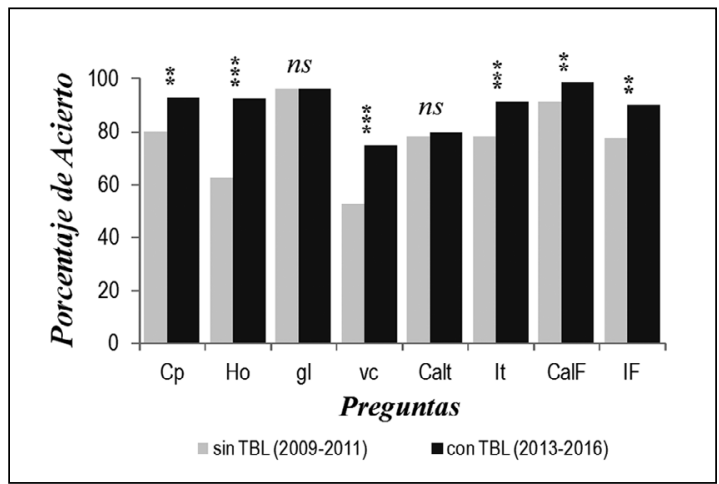

Figura 2. Porcentaje de acierto para las preguntas del Hito 2 , períodos sin y con aprendizaje bajo modalidad TBL. Abreviaciones como en Tabla 1. $\left({ }^{* *} p<0,01 ;{ }^{* *} p<0,001\right.$; ns = no significativo). 
Tabla 2. Resumen de los comentarios realizados por los estudiantes de primer año de medicina, agrupados según impresión general, fase del TBL y otros. Comentarios en paréntesis indican otros conceptos mencionados por los estudiantes asociados al principal

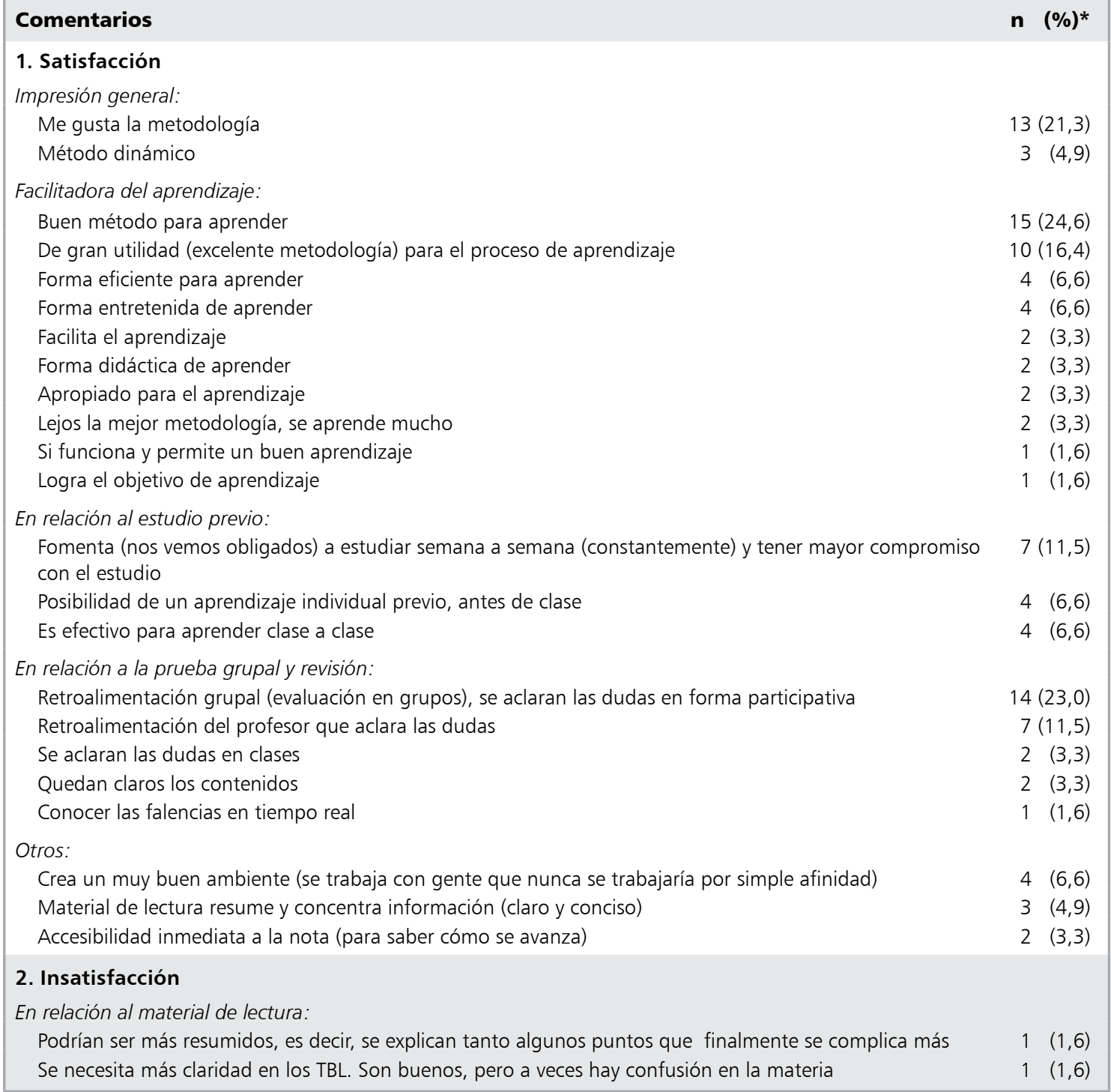

*Porcentaje respecto al total de estudiantes que realizaron comentarios sobre la metodología TBL $(n=61)$. Nota: Los estudiantes mencionaron en sus comentarios de 1 a 4 conceptos.

\section{Discusión}

El aprendizaje basado en equipo, TBL, es una metodología que desafía al estudiante a ser proactivo, que promueve el aprendizaje colaborativo y mejora el pensamiento crítico $^{25}$, pero también ha demostrado mejorar el aprendizaje significativo mejorando el desempeño de los estudiantes en comparación a la clase tradicional ${ }^{9,26,27}$. Nuestros resultados mostraron un mejor desempeño de los estudiantes de medicina en el estudio de la estadística con metodología TBL evidenciado después de un semestre cuando se evaluó una prueba estadística de mayor complejidad (Hito 2). 
El buen rendimiento de los estudiantes observado en ambos períodos en el Hito 1 y respectiva prueba de cátedra sugiere que en materias de baja complejidad el TBL no tendría mayores ventajas frente a la clase magistral, aunque las conductas de entrada de los estudiantes de primer año de medicina incluyen conocimientos de estadística descriptiva, distribución normal y Z. Si bien el diseño del estudio no permite discriminar entre estrategia docente versus conducta de entrada, la percepción de los estudiantes sí permite establecer una ventaja de la metodología TBL sobre la clase magistral al ser considerada entretenida y facilitadora del aprendizaje, además de reafirmar los conceptos de proactividad y aprendizaje colaborativo, todo esto mejorando el ambiente en el aula y abordando la bioestadística de manera más amigable. Lo anterior es ampliamente evidenciado en las distintas áreas de la medicina ${ }^{26-29}$, pero escasamente en cursos de estadísticas en carreras de las ciencias de la salud ${ }^{23-24}$.

El desempeño de los estudiantes en el Hito 2 permite identificar áreas dónde la estrategia TBL contribuyó a un mejor aprendizaje. Mejoró lo cognitivo, reteniendo de mejor manera los conceptos incluidos en el análisis estadístico y en el cómo identificar correctamente los valores críticos para un tamaño de muestra determinado, considerando la unilateralidad o bilateralidad de la prueba. Mejoró el pensamiento lógico, discerniendo con mayor precisión la pregunta de investigación y, por tanto, estableciendo de manera correcta las respectivas hipótesis estadísticas. Además, mejoró el pensamiento crítico dado que, al entender y ordenar de mejor manera la información disponible, la interpretación de la prueba estadística fue adecuada $y$, en consecuencia, respondieron correctamente a la pregunta de investigación. El análisis de las preguntas, también, mostró que los ejercicios descritos paso a paso en el material de lectura no produjeron una mejora en el cálculo del estadístico $t$, siendo las sesiones de ejercicios las mismas en ambos períodos. Esto es un punto a mejorar. No obstante, sí mejoró el cálculo del estadístico $\mathrm{F}$ que es un procedimiento más simple.

Exponer al estudiante a sucesivas pruebas de baja complejidad mejora la comprensión y retención ${ }^{30-32}$, estrategia utilizada por la metodología TBL durante el desarrollo de una sesión (prueba individual y prueba grupal) como en sesiones concatenadas que terminan en una prueba de cátedra.
Esta exposición, junto con mejorar el desempeño individual, fue valorado por los estudiantes porque los mantuvo en un ritmo constante de estudio de baja intensidad que los ordenó y restó presión.

La experiencia ganada año a año por el docente pudo tener un efecto importante en el desempeño de los estudiantes e incidir en la buena valoración que en este estudio se hace de la metodología TBL, factor que pudo estar presente pero difícil de aislar. Sin embargo, nuestro equipo lo minimiza porque la cátedra de estas asignaturas ha estado liderada por el mismo docente desde el año 2004, porque la mejora es evidenciada inmediatamente en la primera evaluación de MI-II que es un curso más complejo donde el rendimiento siempre había sido notoriamente menor que MI-I y por el ambiente favorable que continuó con la práctica del TBL en MI-II. Sumado a que el material de lectura y las pruebas individuales/grupales no han sufrido cambios en contenido ni estructura, nuestros resultados nos permiten establecer que la metodología TBL mejoró el desempeño de los estudiantes de medicina en el estudio de la estadística favoreciendo la movilización de recursos cognitivos. La consecuencia inmediata es que el estudiante puede abordar de mejor manera la literatura científica en que se basan la mayoría de las cátedras, propiciar la investigación científica y, a futuro, contribuir a una labor profesional con una mejor base científica.

Los resultados de la metodología TBL cumplen con los requisitos de los dos primeros niveles del modelo de evaluación de programas de Kirkpatrik para medir el impacto de la estrategia implementada, esto es, reacción (valoración positiva de la metodología) y aprendizaje (aumento significativo del desempeño) ${ }^{33}$, y avala su replicación en cursos similares. Sin embargo, como el desempeño y disposición hacia la estadística también responde a habilidades y/o conocimientos adquiridos previamente en matemáticas ${ }^{34-37}$, la metodología TBL no necesariamente pueda ser valorada de igual manera en todas las carreras de la salud ya que las conductas de ingreso de los estudiantes (habilidades y/o conocimiento en matemáticas) difieren entre ellas. Este es un aspecto importante a considerar y requiere ser abordado.

Agradecimientos: A la Dra. Claudia Behrens de la Oficina de Educación Médica de la Facultad de Medicina, Universidad Católica del Norte, por su 
apoyo y revisión crítica del manuscrito. Al revisor anónimo por sus comentarios y sugerencias que mejoraron la versión final.

\section{Referencias}

1. Clouet-Huerta DE, Correa K. Investigación médica en pregrado: ¿qué está sucediendo en Chile? Rev Med Chile 2014; 142 (11): 1488-90.

2. Araos-Baeriswyl E, Moll-Manzur C. ¿Cuánto investigan los estudiantes de medicina en pregrado? Rev Med Chile 2015; 143: 1358-9.

3. Clouet-Huerta DE, Correa K. Vinculando la investigación científica con la formación de pregrado en carreras de la salud. Rev Med Chile 2017; 145: 549-50.

4. Djulbegovic B, Guyatt GH. Progress in evidence-based medicine: a quarter century on. Lancet 2017; 390: 41523.

5. Freeman S, Eddy SL, McDonough M, Smith MK, Okoroafor N, Jordt H, Wenderoth MP. Active learning increases student performance in science, engineering, and mathematics. Proceedings of the National Academy of Sciences 2014; 111: 8410-5.

6. Garfield J. Teaching Statistics Using Small-Group Cooperative Learning. Journal of Statistics Education 1993; 1 (1), DOI: 10.1080/10691898.1993.11910455.

7. Giraud G. Cooperative Learning and Statistics Instruction. Journal of Statistics Education 1997; 5 (3), DOI: 10.1080/10691898.1997.11910598.

8. Roseth CJ, Garfield JB, Ben-Zvi D. Collaboration in Learning and Teaching Statistics. Journal of Statistics Education 2008; 16 (1), DOI: 10.1080/10691898.2008.11889557.

9. Kamei RK, Cook S, Puthucheary J, Starmer F. 21st Century Learning in Medicine: Traditional Teaching versus Team-based Learning. Med Sci Educ 2012; 22 (2): 57-64.

10. James S, Inscoe HD. Report on the 17th Annual Meeting of the Team-Based Learning Collaborative. Med Sci Educ 2018; 28 (2): 457-8.

11. Parmelee D, Michaelsen LK, Cook S, Hudes PD. Team-based learning: A practical guide 2012. AMEE Guide No. 65. Med Teach, 2012; 34: e275-e287.

12. Moraga D, Soto J. TBL-Aprendizaje Basado en Equipos. Estudios Pedagógicos 2016; 42 (2): 437-47.

13. Michaelsen LK, Watson WE, Cragin JP, Fink LD. Team learning: A potential solution to the problems of large classes. Exchange: The Organizational Behavior Teaching Journal 1982; 7: 13-22.

14. Haidet P, Levine RE, Parmelee DX, Crow S, Kennedy
F, Kelly PA, et al. Guidelines for Reporting Team-Based Learning Activities in the Medical and Health Sciences Education Literature. Acad Med 2012; 87 (3): 292-9.

15. Huggett KN, White MD, Knoop FC, Eno CA, Cullen DM. Team-Based Learning in Anatomy Lab: Promoting Active Learning and Professionalism. Med Sci Educ 2016; 26: 5-6.

16. Hernández M, Giannini J, Alston S, Vasauskas A. Use of Live-Stream and Pre-recorded Simulation in the Individual Readiness Assessment Test During TBL, a Novel Approach. Med Sci Educ 2017; 27: 15-19.

17. Alimoglu MK, Yardım S, Uysal H. The effectiveness of TBL with real patients in neurology education in terms of knowledge retention, in-class engagement, and learner reactions. Adv Physiol Educ 2017; 41: 38-43.

18. Richardson-Hatcher A, Hazzard M, Bentley C, Gazave C, Greenlee T, Brueckner-Collins J. Team-Based Learning in a 3D Online Environment. Med Sci Educ 2014; 24: 11-14.

19. Gazave CM, Hatcher AR. Evaluating the Use of Second Life $^{\mathrm{TM}}$ for Virtual Team-Based Learning in an Online Undergraduate Anatomy Course. Med Sci Educ 2017; 27: 217-27.

20. Vogeltanz-Holm N, Olson LM, Borg KE, Hill TM. Implementation and Evaluation of a Team-Based Learning Approach Within a Hybrid Problem-Based Learning Medical Education Curriculum. Med Sci Educ 2014; 24: 125-33.

21. Burgess A, Roberts C, Ayton T, Mellis C. Implementation of modified team-based learning within a problem based learning medical curriculum: a focus group study. BMC Medical Education 2018; 18: 74-80.

22. Clair KS, Chihara L. Team-Based Learning in a Statistical Literacy Class. Journal of Statistics Education 2012; 20: 1. DOI: 10.1080/10691898.2012.11889633.

23. Hagen B, Awosoga O, Kellett P, Dei SO. Evaluation of undergraduate nursing students' attitudes towards statistics courses, before and after a course in applied statistics. Nurse Education Today 2013; 33: 949-55.

24. Tavares Paes A. Perspectives on teaching biostatistics for undergraduate medical students using Team-Based Learning. Satellite Papers of The International Association for Statistical Education 2015. https://iase-web.org/ documents/papers/sat2015/IASE2015\%20 Satellite\%20 51_PAES.pdf

25. Michaelsen LK, Sweet M. Team-based learning. New directions for Teaching and Learning 2011; 128: 41-51.

26. Reimschisel T, Herring AL, Huang J, Minor TJ. A systematic review of the published literature on team-based learning in health professions education, Medical Teacher 2017; 39 (12): 1227-37. 
27. Chen M, Ni C, Hu Y, Wang M, Liu L, Ji X, et al. Meta-analysis on the effectiveness of team-based learning on medical education in China. BMC Medical Education 2018; 18: 77-87.

28. Burgess AW, McGregor DM, Mellis CM. Applying Established Guidelines to Team-Based Learning Programs in Medical Schools: A Systematic Review. Acad Med 2014; 89 (4): 678-88.

29. Obad AS, Peeran AA, Shareef MA, Alsheikh WJ, Kalagi DA, AlAmodi AA, et al. Assessment of first-year medical students' perceptions of teaching and learning through team-based learning sessions. Adv Physiol Educ 2016; 40: 536-42.

30. Larsen DP, Butler AC, Roediger III HL. Repeated testing improves long term retention relative to repeated study: a randomized controlled trial. Medical Education 2009; 43 (12): 1174-81.

31. Chang EK, Wimmers PF. Effect of Repeated/Spaced Formative Assessments on Medical School Final Exam Performance. Health Professions Education 2017; 3 (1): 32-7.
32. Spreckelsen C, Juenger J. Repeated testing improves achievement in a blended learning approach for risk competence training of medical students: results of a randomized controlled trial. BMC Medical Education 2017; 17: 177-86.

33. Kirkpatrick JD, Kirkpatrick WK. Kirkpatrick's Four Levels of Training Evaluation. Virginia, USA: ATD Press; 2016. 200 pp.

34. Chiesi F, Primi C. Cognitive and non-cognitive factors related to students' statistic achievement. Stat Educ Res Jour 2010; 9: 6-26.

35. Hannigan A, Hegarty AC, McGrath D. Attitudes towards statistics of graduate entry medical students: the role of prior learning experiences. BMC Med Educ 2014; 14: 70-6.

36. Stanisavljevic D, Trajkovic G, Marinkovic J, Bukumiric Z, Cirkovic A, Milic N (2014) Assessing Attitudes towards Statistics among Medical Students: Psychometric Properties of the Serbian Version of the Survey of Attitudes Towards Statistics (SATS). PLoS ONE 9 (11): $1-7$. 